\title{
THE SINGULAR COHOMOLOGY OF THE INVERSE LIMIT OF A POSTNIKOV TOWER IS REPRESENTABLE
}

\author{
JERZY DYDAK AND ROSS GEOGHEGAN
}

\begin{abstract}
Let $X_{1} \stackrel{f_{1}}{\longleftarrow} X_{2} \stackrel{f_{2}}{\longleftarrow} \cdots$ be an inverse sequence of spaces and maps satisfying (i) each $X_{n}$ has the homotopy type of a CW complex, (ii) each $f_{n}$ is a Hurewicz fibration, and (iii) the connectivity of the fiber of $f_{n}$ goes to $\infty$ with $n$. Let $\hat{X}$ be the inverse limit of the sequence. It is shown that the natural homomorphism $\breve{H}^{k}(\hat{X}, G) \rightarrow H^{k}(\hat{X}, G)$ (from Cech cohomology to singular cohomology, with ordinary coefficient module $G$ ) is an isomorphism for all $k$. It follows that $\lim _{n}\left[X_{n}, K(G, k)\right] \cong[\hat{X}, K(G, k)]$ for any EilenbergMac Lane space $K(G, k)$. It is also shown that, except in trivial cases, $\hat{X}$ does not have the homotopy type of a $\mathrm{CW}$ complex.
\end{abstract}

1. Statements. We understand that certain homotopy theorists have use for the theorem which forms the title of this note. The natural theorem (Theorem A) turns out to be somewhat more general. We also prove a companion result (Theorem $\mathrm{B})$ to show that Theorem $\mathrm{A}$ is not frivolous.

We will denote singular cohomology by $H^{*}$ and Cech cohomology based on normal (三 numerable) open covers by $\breve{H}^{*}$. $\check{H}^{*}$ is the representable theory; i.e. when $Z$ is a space and $G$ is an abelian group, there is a natural bijection $\operatorname{Ho}(Z, K(G, k)) \rightarrow$ $\breve{H}^{k}(Z ; G)$. This is standard knowledge when $Z$ is a CW complex. When $Z$ is an arbitrary space the explanation (which has long been known) can be sketched as follows:

$$
\begin{aligned}
\operatorname{Ho}(Z, K(G, k)) & \cong \operatorname{Sh}(Z, K(G, k)) \cong \operatorname{pro}-\operatorname{Ho}\left(\left\{Z_{\alpha}\right\}, K(G, k)\right) \\
& \cong \underset{\alpha}{\lim } \operatorname{Ho}\left(Z_{\alpha}, K(G, k)\right) \cong \underset{\alpha}{\lim } H^{k}\left(Z_{\alpha} ; G\right) \equiv \check{H}^{k}(Z ; G) .
\end{aligned}
$$

[The notation here is: $C(A, B)$ is the set of morphisms from $A$ to $B$ in the category $C$; Ho=homotopy; $\mathrm{Sh}=$ shape; pro- $C$ is the pro-category of inverse systems in $C$; $\left\{Z_{\alpha}\right\}$ in pro-Ho is the Cech expansion of $Z$ (see [MS]) for details.]

THEOREM A. Let

$$
X_{1} \stackrel{f_{1}}{\longleftarrow} X_{2} \stackrel{f_{2}}{\longleftarrow} \cdots
$$

be a sequence of Hurewicz fibrations, where each $X_{n}$ has the homotopy type of a CW complex, and for any $N$, all but finitely many of the $f_{n}$ have $N$-connected fibers. Let $G$ be an abelian group and let $\hat{X}$ be the inverse limit of the sequence. Then the natural homomorphism $\breve{H}^{k}(\hat{X} ; G) \rightarrow H^{k}(\hat{X} ; G)$ is an isomorphism for all $k$.

Received by the editors July 30, 1985.

1980 Mathematics Subject Classification (1985 Revision). Primary 55S45, 55P55, 54E60.

Key words and phrases. Cech cohomology, Postnikov system, Homotopy type of CW complex. 
COROLLARY. There is a natural isomorphism

$$
\underset{n}{\lim } \operatorname{Ho}\left(X_{n}, K(G, k)\right) \cong \operatorname{Ho}(\hat{X}, K(G, k)) \text {. }
$$

Theorem A might lead the reader to ask if, perhaps, $\hat{X}$ has the homotopy type of a CW complex. Apart from trivial cases, the answer is NO:

THEOREM B. With hypotheses as in Theorem A, $\hat{X}$ has the homotopy type of a $C W$ complex if and only if all but finitely many of the $f_{n}$ are homotopy equivalences.

The reader who wants a quick idea of the proof of Theorem A should read the statements of Lemmas 1-6 and "Proof of Theorem A" which follows them.

We thank Gaunce Lewis for posing the problem to us.

2. Proofs. We break up the proof of Theorem $A$ into lemmas, which we begin by listing.

Recall that the space $Z$ is $\mathrm{LC}^{n}$ if for each $z \in Z$ and each neighborhood $U$ of $z$, there is a smaller neighborhood $V$ of $z$ such that if $i: V \rightarrow U$ is inclusion, then $i_{\#}: \pi_{k}(V, z) \rightarrow \pi_{k}(U, z)$ is trivial for $k \leq n . Z$ is $\mathrm{LC}^{\infty}$ if it is $\mathrm{LC}^{n}$ for all $n$. Note that $Z$ is $\mathrm{LC}^{0}$ if and only if $Z$ is locally path connected (path components of open sets are open).

LEMMA 1. Let

$$
X_{1} \stackrel{f_{1}}{\longleftarrow} X_{2} \stackrel{f_{2}}{\longleftarrow} \cdots
$$

be a sequence of Serre fibrations, where each $X_{n}$ is metrizable and $L C^{\infty}$, and the connectivity of $f_{n}$ goes to $\infty$ with $n$. Then the inverse limit, $\hat{X}$, is metrizable and $L C^{\infty}$.

LEMMA 2. If the connected space $Z$ is metrizable and $L C^{\infty}$, then the natural homomorphism $\breve{H}^{k}(Z ; G) \rightarrow H^{k}(Z, G)$ is an isomorphism for all $k$ and all abelian groups $G$.

Recall that the metrizable space $Z$ is an absolute neighborhod retract for metrizable spaces (abbreviated ANR) if whenever $Z$ is embedded as a closed subset of a metrizable space $M, Z$ is a retract of one of its neighborhoods in $M$.

LEMMA 3. Every $C W$ complex has the homotopy type of an ANR.

LEMMA 4. Every $A N R$ is $L C^{\infty}$.

LEMMA 5. Let $f: Y \rightarrow Z$ be a map and let $P_{f}=\left\{(y, \omega) \in Y \times Z^{I} \mid \omega(0)=f(y)\right\}$. If $Y$ and $Z$ are $A N R$ 's, then $P_{f}$ is an ANR. Moreover $f$ factorizes as $p \circ s$, where $s: Y \rightarrow P_{f}$ is a homotopy equivalence and $p: P_{f} \rightarrow Z$ is a Hurewicz fibration.

LEMMA 6. Let the following diagram commute:

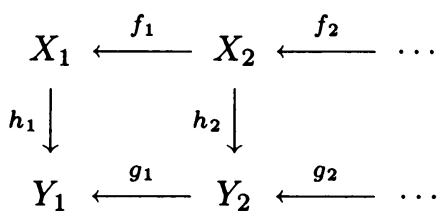


where each $f_{n}$ and each $g_{n}$ is a Hurewicz fibration and each $h_{n}$ is a homotopy equivalence. Then the inverse limit $\hat{h}: \hat{X} \rightarrow \hat{Y}$ is a homotopy equivalence.

Proof of TheOREM A (USING Lemmas 1-6). Wlog, assume each $X_{n}$ is path connected. Then $\hat{X}$ is path connected [MS, p. 178]. By Lemmas 3 and 5 we can get a commutative diagram (*), as in Lemma 6, such that each $Y_{n}$ is an ANR, each $g_{n}$ is a Hurewicz fibration, and each $h_{n}$ is a homotopy equivalence. By Lemma $6, \hat{Y} \equiv \lim \left\{Y_{1} \stackrel{g_{1}}{\longleftarrow} Y_{2} \stackrel{g_{2}}{\longleftarrow} \cdots\right\}$ is path connected, and it is enough to prove the theorem for $\hat{Y}$. By Lemmas 1 and $4, \hat{Y}$ is metrizable and LC ${ }^{\infty}$. Therefore Lemma 2 implies $\breve{H}^{k}(\hat{Y} ; G) \rightarrow H^{k}(\hat{Y} ; G)$ is an isomorphism.

Now we will give proofs or references for Lemmas $1-6$.

PROOF OF LEMMA 1. [This is surely a folk theorem, certainly in the compact case.] A basic open neighborhood of $\hat{x} \in \hat{X}$ has the form $f^{-1}\left(U_{m}\right)$, where $f: \hat{X} \rightarrow$ $X_{m}$ is projection and $U_{m}$ is a neighborhood of $x_{m} \equiv f(\hat{x})$ in $X_{m}$. Since $X_{m}$ is $\mathrm{LC}^{0}$, it is locally path connected, so we may choose $U_{m}$ path connected. Fix $k$, and let $m$ be such that $f_{n}$ has $(k+1)$-connected fibers for all $n \geq M$. Let $V_{m} \subset U_{m}$ be a path connected neighborhood of $x_{m}$ such that $\pi_{i}\left(V_{m}, x_{m}\right) \rightarrow \pi_{i}\left(U_{m}, x_{m}\right)$ is trivial for $i \leq k$. Now $\pi_{i}\left(V_{n}, x_{n}\right) \rightarrow \pi_{i}\left(V_{m}, x_{m}\right)$ and $\pi_{i}\left(U_{n}, x_{n}\right) \rightarrow \pi_{i}\left(U_{m}, x_{m}\right)$ are isomorphisms for $i \leq k$ and $n \geq m$, where $U_{n}$ and $V_{n}$ are the preimages of $U_{m}$ and $V_{m}$ in $X_{n}$. Hence $\pi_{i}\left(V_{n}, x_{n}\right) \rightarrow \pi_{i}\left(U_{n}, x_{n}\right)$ is trivial for $i \leq k$. Thus $\lim _{n} \pi_{i}\left(V_{n}, y_{n}\right) \rightarrow \lim _{n} \pi_{i}\left(U_{n}, y_{n}\right)$ is trivial for $i \leq k$.

There is a well-known natural exact sequence of pointed sets (indeed of groups when $i \geq 1$ ):

$$
0 \rightarrow \underset{n}{\lim ^{1}} \pi_{i+1}\left(U_{n}, x_{n}\right) \rightarrow \pi_{i}(\hat{U}, \hat{x}) \rightarrow \underset{n}{\lim } \pi_{i}\left(U_{n}, x_{n}\right) \rightarrow 0,
$$

where $\hat{U}=f^{-1}\left(U_{m}\right)$ (see, for example, [MS, p. 178]). In our case, the lim ${ }^{1}$ terms vanish, so $\pi_{i}(\hat{U}, \hat{x})$ is naturally isomorphic to $\lim \pi_{i}\left(U_{n}, x_{n}\right)$ for all $i$; the case $i=0$ causes no trouble since $U_{m}$, hence each $U_{n}^{n}$, is path connected. Similar remarks apply when $V$ replaces $U$. By naturality, $\pi_{i}(\hat{V}, \hat{x}) \rightarrow \pi_{i}(\hat{U}, \hat{x})$ is zero for $i \leq k$. Thus $\hat{X}$ is $\mathrm{LC}^{k}$. Since $k$ was arbitrary, $\hat{X}$ is $\mathrm{LC}^{\infty}$. Since $\hat{X} \subset \prod_{n=1}^{\infty} X_{n}, \hat{X}$ is also metrizable.

PROOF OF LEMMA 2. $Z$ is connected and $\mathrm{LC}^{0}$, hence path connected. Pick a base point $z \in Z$. Let $\left\{\left(Z_{\alpha}, z_{\alpha}\right)\right\}$ be the pointed Cech expansion of $(Z, z)$, i.e. the inverse system of nerves of all "pointed" normal (三numerable) open covers of $Z$, whose bonding morphisms in the homotopy category are induced by refinement (see [MS, p. 329]). Let $Z_{\alpha}^{n}$ denote the $n$-skeleton of $Z_{\alpha}$. Fix $k$. Since $Z$ is metrizable and $\mathrm{LC}^{k+1}$, the proof of Theorem 8.9 of $[\mathrm{Dy}]$ implies that there is a unique morphism $q$ of pro- $\mathrm{Ho}_{0}$ making the following diagram commute:

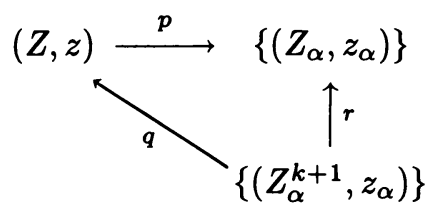

Here, $\mathrm{Ho}_{0}$ is the pointed homotopy category of spaces, $p$ is the canonical morphism, and $r$ is induced by inclusion. Hence [MS, p. 222] $\left\{\pi_{i}\left(Z_{\alpha}, z_{\alpha}\right)\right\}$ is stable for $i \leq k$. 
By Corollary 2.2 of $[\mathbf{K S}]$, we see that $p_{\#}: \pi_{i}(Z, z) \rightarrow \varliminf_{\lim _{\alpha}} \pi_{i}\left(Z_{\alpha}, z_{\alpha}\right)$ is an isomorphism for $i \leq k$. The last two sentences imply $p_{\#}:{\overleftarrow{\pi_{i}(Z, z)}}^{\alpha}, z\left\{\pi_{i}\left(Z_{\alpha}, z_{\alpha}\right)\right\}$ is an isomorphism in pro-Groups when $1 \leq i \leq k$ and of pro-(Pointed Sets) when $i=0$. Since $r=p \circ q, p_{\#}$ is also an epimorphism when $i=k+1$ [MS, p. 112]. By the relative pro-Hurewicz Theorem [MS, p. 140], $p_{*}: H_{i}(Z) \rightarrow\left\{H_{i}\left(Z_{\alpha}\right)\right\}$ is an isomorphism of pro-Groups for $i \leq k$. (Note; the theorem in [MS] requires polyhedra, so we must first apply the "geometric realization of the singular complex" functor. And it requires that pro- $\pi_{0}$ be trivial: this is where we use the fact that $Z$ is path connected.) Applying Hom and Ext to $p_{*}$, we get appropriate isomophisms in the dual category, inj-Groups, of direct systems of abelian groups. Thus, applying the (functorial) Universal Coefficient Theorem, and taking direct limits, we get a commutative diagram for each $i \leq k$ :

$$
\begin{aligned}
& 0 \rightarrow \underset{\alpha}{\lim } \operatorname{Ext}\left(H_{i-1}\left(Z_{\alpha}\right), G\right) \rightarrow \underset{\alpha}{\lim _{\vec{\alpha}}} H^{i}\left(Z_{\alpha} ; G\right) \rightarrow \underset{\alpha}{\lim } \operatorname{Hom}\left(H_{i}\left(Z_{\alpha}\right), G\right) \rightarrow 0 \\
& \downarrow \\
& 0 \rightarrow \operatorname{Ext}\left(H_{i-1}(Z), G\right) \quad \rightarrow \quad H^{i}(Z ; G) \quad \rightarrow \quad \operatorname{Hom}\left(H_{i}(Z), G\right) \quad \rightarrow 0
\end{aligned}
$$

Here the vertical arrows are induced by $p$, and the outer arrows are isomorphisms, hence also the middle arrow.

PROOF OF LEMMA 3. Every CW complex has the homotopy type of a simplicial complex with the weak topology. To see this, simplicially approximate the attaching maps, by induction on skeleta, and triangulate. That every simplicial complex with the weak topology has the homotopy type of an ANR is a classical theorem: for a complete modern proof see [MS, p. 319]. The steps are: (i) the metric topology is homotopy equivalent to the weak topology, and (ii) the metric topology gives an ANR.

ProOF OF LEMMA 4. See [MS, p. 40]. We sketch an alternative proof. Embed the ANR $Z$ as a closed subset of its convex hull in the metric linear space of bounded maps from $Z$ to $\mathbf{R}$ (sup metric) (see [D, p. 286]). Since $Z$ is a neighborhod retract of a locally convex space, $Z$ is clearly $L^{\infty}$.

Proof of LemMa 5. For the factorization, see [S, p. 99]. The fact that $P_{f}$ is an ANR is an easy exercise, or see [H, p. 188].

Proof of LEMMA 6. This is due to Edwards and Hastings [EH, p. 95]. For a self-contained two-page proof, see $[\mathbf{G}]$.

PROOF OF COROLLARY. The exact sequence used in the proof of Lemma $1 \mathrm{im}-$ plies $\pi_{k}(\hat{X}, \hat{x}) \rightarrow \lim _{n} \pi_{k}\left(X_{n}, x_{n}\right)$ is an isomorphism (where again wlog we assume $X_{1}$ is path connected). But $\left\{\pi_{k}\left(X_{n}, x_{n}\right)\right\}$ is stable. So $\pi_{k}(\hat{X}, \hat{x}) \rightarrow\left\{\pi_{k}\left(X_{n}, x_{n}\right)\right\}$ is an isomorphism in pro-Groups. It follows, as in the proof of Lemma 2, that $\lim _{n} H^{k}\left(X_{n} ; G\right) \rightarrow H^{k}(\hat{X} ; G)$ is an isomorphism for all $k$. This, together with $\overrightarrow{T h e o r e m ~ A}$, gives the Corollary.

We now turn to Theorem B.

A space $Z$ is semilocally contractible if each point of $Z$ has a neighborhood which contracts in $Z$. $Z$ is locally contractible if for each $z \in Z$ and each neighborhood $U$ of $z$, there is a smaller neighborhood $V$ of $z$ such that $V \hookrightarrow U$ is homotopically trivial. If $Z$ is homotopy equivalent to a locally contractible space, then $Z$ is semilocally contractible (this is an easy exercise). Since ANR's are locally contractible ([MS, 
p. 40] or see the proof of Lemma 4), Lemma 3 implies that semilocal contractibility is a necessary condition for having the homotopy type of a $\mathrm{CW}$ complex.

PROOF OF THEOREM B. Let $T$ be the locally compact CW complex obtained from $[0, \infty) \amalg \coprod_{n=1}^{\infty} S^{n}$ by identifying the point $u_{n} \in S^{n}$ with $n \in[0, \infty)$ for all $n$. Thus $T$ consists of higher and higher-dimensional lightbulbs suspended from an infinite electric wire. Let $T^{+}$be the one-point compactification of $T$; think of the new point as $\infty$, and high-dimensional bulbs as being small and near $\infty$. Let infinitely many $f_{n}$ not be homotopy equivalences. Wlog, assume $f_{n \#}: \pi_{n}\left(X_{n+1}, x_{n+1}\right) \rightarrow \pi_{n}\left(X_{n}, x_{n}\right)$ has a nonzero element in its kernel, represented by $g_{n+1}^{n}:\left(S^{n}, u_{n}\right) \rightarrow\left(X_{n+1}, x_{n+1}\right)$, while $f_{n}$ induces isomorphisms on $\pi_{i}$ when $i<n$. Let $g_{r}^{n}:\left(S^{n}, u_{n}\right) \rightarrow\left(X_{r}, x_{r}\right)$ represent the corresponding element in $\pi_{n}\left(X_{r}, x_{r}\right)$ for all $r$. This means $f_{r} \circ g_{r+1}^{n} \simeq g_{r}^{n}$ rel $u_{n}$. In particular, choose $g_{r}^{n}$ to be constant (at $x_{r}$ ) when $r \leq n$. Define $G_{r}: T^{+} \rightarrow X_{r}$ by $G_{r}([0, \infty])=x_{r}$ and $G_{r} \mid S^{n}=g_{r}^{n}: S^{n} \rightarrow X_{r}$. Since $g_{r}^{n}$ is constant when $r \leq n, G_{r}$ is continuous, Moreover $f_{r} \circ G_{r+1} \simeq G_{r}$. Since the $f_{n}$ are fibrations, we can alter the $G_{r}$ within their homotopy classes so that $f_{r} \circ G_{r+1}=G_{r}$ for all $r$. Then there is an induced map $G: T^{+} \rightarrow \hat{X}$. Suppose $\hat{X}$ were semilocally contractible. Then, for all but finitely many $n, G \mid S^{n}$ would be homotopically trivial, since a neighborhood of $G(\infty)$ would contract in $\hat{X}$. This would contradict the fact that $\left[g_{n+1}^{n}\right] \neq 0 \in \pi_{n}\left(X_{n+1}, x_{n+1}\right)$ for all $n$. Thus $\hat{X}$ does not have CW homotopy type.

Conversely, let all but finitely many $f_{n}$ be homotopy equivalences: wlog, let all be. Apply Lemma 6 with each $Y_{n}=X_{1}$, each $g_{n}=$ identity, and $h_{n}=f_{1} \circ \cdots \circ f_{n-1}$, to conclude that $\hat{X}$ has the homotopy type of $X_{1}$.

3. A variant. A space is hereditarily paracompact if every subset (equivalently, every open subset) is paracompact [LW, pp. 204-205]. CW complexes are hereditarily paracompact [LW, p. 56]. The proof of Theorem A can be shortened, if one knows that each $X_{n}$ is hereditarily paracompact. One needs the following lemma.

LEMMA 7. If $\hat{X}=\lim \left\{X_{1} \leftarrow X_{2} \leftarrow \cdots\right\}$, where each $X_{n}$ is hereditarily paracompact, then $\hat{X}$ is paracompact (indeed, hereditarily).

ProOF. Let $\mathcal{U}$ be an open cover of $\hat{X}$. Refining $U$ if necessary, assume each element of $\mathcal{U}$ has the form $p_{n}^{-1}\left(U_{n}\right)$ for some $n$, where $p_{n}: \hat{X} \rightarrow X_{n}$ is projection. For each $n$, let $U_{n}$ consist of those members of $U$ which have the form $p_{n}^{-1}\left(U_{n}\right)$. Then $U=\bigcup_{n=1}^{\infty} U_{n}$. For each $n, \bigcup p_{n}\left(\mathcal{U}_{n}\right)$ is open in $X_{n}$, hence is paracompact, so $p_{n}\left(U_{n}\right)$ has a locally finite refinement, hence $U_{n}$ has a locally finite refinement. Thus $\mathcal{U}$ has a $\sigma$-locally finite refinement. Now $\hat{X}$ is clearly regular [D, p. 142], so $\hat{X}$ is paracompact $[\mathbf{K}$, p. 156$]$.

One also needs Lemma 2 for paracompact spaces. The proof of this is similar to that of Theorem 8.9 of [Dy], and details are left to the interested reader. This, together with Lemma 7 and Lemm 1, gives the desired result.

\section{REFERENCES}

[D] J. Dugundji, Topology, Allyn and Bacon, Boston, Mass., 1966.

[Dy] J. Dydak, The Whitehead and the Smale theorems in shape theory, Dissertationes Math. 156 (1979), 1-55. 
[EH] D. A. Edwards and H. M. Hastings, Cech and Steenrod homotopy theories with applications to geometric topology, Lecture Notes in Math., vol. 542, Springer-Verlag, Berlin, 1976.

[G] R. Geoghegan, The inverse limit of homotopy equivalences between towers of fibrations is a homotopy equivalence-a simple proof, Topology Proc. 4 (1979), 99-101.

[H] S.-T. Hu, Theory of retracts, Wayne State Univ. Press, Detroit, Mich., 1965.

[K] J. L. Kelley, General topology, Van Nostrand, Princeton, N.J., 1955.

[KS] G. Kozlowski and J. Segal, Local behavior and the Vietoris and Whitehead theorems in shape theory, Fund. Math. 99 (1978), 213-225.

[LW] A. T. Lundell and S. Weingram, The topology of $C W$ complexes, Van Nostrand Reinhold, New York, 1969.

[MS] S. Mardešic and J. Segal, Shape theory, North-Holland, Amsterdam, 1982.

[S] E. Spanier, Algebraic topology, McGraw-Hill, New York, 1966.

Department of Mathematics, University of Tennessee, Knoxville, TenNESSEE 37996

Department of MATHEMATICS, State UNiversity OF NEW YoRK AT BinghamTON, BINGHAMTON, NEW YORK 13901 